\title{
Depression of Systolic and Diastolic Myocardial Reserve during Atrial Pacing Tachycardia in Patients with Dilated Cardiomyopathy
}

\author{
Marc D. Feldman, James D. Alderman, Julian M. Aroesty, Henry D. Royal, James J. Ferguson, Robert M. Owen, \\ William Grossman, and Raymond G. McKay \\ Charles A. Dana Research Institute and Harvard-Thorndike Laboratory, Cardiovascular Division, Department of Medicine, \\ Beth Israel Hospital and Harvard Medical School, Boston, Massachusetts 02215
}

\begin{abstract}
Previous reports have shown that increases in heart rate may result in enhanced left ventricular (LV) systolic and diastolic performance. To assess whether this phenomenon occurs in the presence of depressed $\mathrm{LV}$ function, the effects of pacing on $\mathrm{LV}$ pressure and volume were compared in seven patients with dilated cardiomyopathy ( $\mathrm{LV}$ ejection fraction $0.19 \pm 0.11)$ and six patients with no or minimal coronary artery disease (LV ejection fraction $0.69 \pm 0.11$ ). Patients with normal $L V$ function demonstrated significant increases in $L V$ peak-positive $\mathrm{dP} / \mathrm{d} t$, $L V$ end-systolic pressure-volume ratio, $L V$ peak filling rate, and a progressive leftward and downward shift of their pressure-volume diagrams, compatible with increased contractility and distensibility in response to pacing tachycardia. There was no change in $L V$ peak-negative $\mathrm{d} P / \mathrm{d} t$ or tau. Patients with dilated cardiomyopathy, in contrast, demonstrated no increase in either $\mathrm{LV}$ peak-positive $\mathrm{dP} / \mathrm{d} t$ or the end-systolic pressurevolume ratio, and absence of a progressive leftward shift of their pressure-volume diagrams. Moreover, cardiomyopathy patients demonstrated no increase in $L V$ peak-negative $d P / d t$ or $\mathrm{LV}$ peak filling rate and a blunted downward shift of the diastolic limb of their pressure-volume diagrams. Tau, as determined from a derivative method, became abbreviated although never reaching control values.

We conclude that patients with dilated cardiomyopathy may demonstrate little or no significant enhancement in systolic and diastolic function during atrial pacing tachycardia, suggesting a depression of both inotropic and lusitropic reserve.
\end{abstract}

\section{Introduction}

An increase in heart rate produces a positive inotropic stimulus with an increase in peak tension in normal cardiac muscle. This so-called "Treppe" or "staircase" effect was originally documented by Bowditch (1) and subsequently confirmed by Woodworth (2). Koch-Weser (3, 4) and Blinks (4) together later extended this observation to isolated mammalian papil-

A preliminary report of this work was presented at the 35th Annual Scientific Meeting of the American College of Cardiology in Atlanta, Georgia, in March 1986.

Address all correspondence and reprint requests to Dr. Feldman, Cardiac Division, Box 158, Department of Internal Medicine, University of Virginia School of Medicine, Charlottesville, VA 22908.

Received for publication 10 December 1986 and in revised form 31 May 1988.

J. Clin. Invest.

(C) The American Society for Clinical Investigation, Inc.

$0021-9738 / 88 / 11 / 1661 / 09 \quad \$ 2.00$

Volume 82, November 1988, 1661-1669 lary muscle by demonstrating positive inotropic and lusitropic $^{1}$ effects to increasing pacing stimulation rates. Similar observations have been made in both intact animals and humans, with chronotropy-induced increases in isovolumic phase indices (e.g., peak positive $\mathrm{d} P / \mathrm{d} t)(6,7)$ and ejection phase indices (e.g., left ventricular rate of ejection) (8). More recently, our laboratory has documented a leftward and upward shift of the end-systolic pressure-volume relationship in response to atrial pacing, suggesting enhanced contractile function during pacing-induced tachycardia $(9,10)$.

An increase in heart rate may also result in enhanced diastolic performance. In the normal human ventricle, parameters of diastolic function which have been shown to improve during tachycardia include left ventricular peak filling rate (10), peak negative $\mathrm{d} P / \mathrm{d} t(11)$, and posterior wall thinning rate (11). In addition, our laboratory has recently documented a progressive downward and leftward shift of the diastolic limb of the pressure-volume diagram during pacing tachycardia in nonischemic patients (10).

Although the effect of tachycardia in patients with normal left ventricular function has been previously documented, the effect of increasing heart rate on patients with dilated cardiomyopathy and intrinsic myocardial disease is not clear. Work with in vitro animal models of heart failure (12) and with isolated human muscle from patients with congestive heart failure (13) has demonstrated that paired electrical stimulation, an intervention similar to pacing, can demonstrate preservation of a positive inotropic effect. However, clinical trials of paired electrical stimulation in patients with congestive heart failure have been disappointing $(14,15)$.

To further assess the effect of increasing heart rate on ventricular performance in patients with depressed left ventricular function, the purpose of the present study was to examine parameters of systolic and diastolic function during atrial pacing in patients with nonischemic dilated cardiomyopathy. Chronotropy-induced changes in ventricular performance in these patients were compared to a normal control group, consisting of patients with no or minimal coronary artery disease and normal ventricular function.

\section{Methods}

Study group. Atrial pacing with simultaneous hemodynamic monitoring and radionuclide ventriculography was conducted at the time of cardiac catheterization in 13 patients. Group A consisted of seven patients referred for evaluation of congestive heart failure and found to have dilated cardiomyopathy with normal coronary arteries. In this group, there were five men and two women with a mean age of $52 \mathrm{yr}$. These patients were being treated with furosemide $(n=5)$, digoxin $(n$ $=7)$, and captopril $(n=2)$, alone or in combination. Five of the seven patients had electrocardiographic evidence of left ventricular hyper-

1. The term lusitropic refers to relaxation-promoting properties (5). 
trophy. One patient had left bundle branch block, and a second an intraventricular conduction delay. Group B consisted of six control patients referred for evaluation of chest pain syndromes, but found to have mild or no coronary artery disease. In this group there were two men and four women with a mean age of $58 \mathrm{yr}$. These patients were thought to have stable angina pectoris and were being treated with long-acting nitrates $(n=4)$, beta-adrenergic blockers $(n=3)$, and calcium-channel blocking agents $(n=5)$ alone or in combination. All medications in both groups were continued up until the time of cardiac catheterization. No patient had evidence of unstable angina, acute myocardial infarction, valvular heart disease, or significant ventricular ectopy. All patients gave written consent of a protocol approved by the Beth Israel Committee on Clinical Investigations. There were no complications as a result of this study.

Cardiac catheterization and coronary angiography. All patients underwent routine right and left heart catheterization, after administration of a local anesthetic. Right heart catheterizations were performed utilizing 7F flow-directed balloon-tipped catheters (Mansfield Scientific, Mansfield, MA), which were inserted percutaneously into the right internal jugular vein and advanced to the pulmonary artery. Coronary angiography was performed in the routine manner utilizing the Judkins technique from the right femoral artery. Left ventriculography was performed with a pigtail catheter, with cine recording in the right anterior oblique projection. Ionic contrast medium (Angiovist 370, Berlex Laboratories, Wayne, NJ) was used.

After left ventriculography, the left ventricular fluid-filled pigtail catheter was replaced with a high-fidelity micromanometer catheter (Millar Instruments, Houston, TX). Recordings were inscribed by means of an Electronics-for-Medicine VR-12 recorder (Honeywell, Inc., Pleasantville, NY).

Atrial pacing protocol. After the completion of coronary angiography and left ventriculography, a biopolar, flared pacing catheter (AtriPace Flare, Mansfield Scientific) was placed within the right atrium via a percutaneous puncture of the right femoral vein. Approximately 30 min after angiography, baseline measurements were made including micromanometer left ventricular pressure, peak positive and negative $\mathrm{d} P / \mathrm{d} t$, Fick cardiac output, and radionuclide ventriculography.

Pacing tachycardia was subsequently initiated at an intermediate level (baseline heart rate plus 25 beats/min) and later increased to a high level (baseline heart rate plus 50 beats/min). Each stage was maintained for $6 \mathrm{~min}$. If atrioventricular block occurred at the high pacing level, the pacing rate was progressively reduced until the heart block disappeared. In all patients, the high pacing level was at least 40 beats/min above the baseline. At each pacing level, repeat measurements of micromanometer left ventricular pressure, $\mathrm{d} P / \mathrm{d} t$, Fick cardiac output, and radionuclide ventriculography were made.

Calculation of tau. The time constant of left ventricular pressure decay $(T)$ was determined from analysis of left ventricular micromanometer pressure tracings. Recordings were made at a paper speed of $100 \mathrm{~mm} / \mathrm{s}$. Left ventricular pressure was digitized "off-line" using a microcomputer (IBM-AT) and graphics tablet (model 1812, Summagraphics Corp., Fairfield, CT) at 5-ms intervals during the isovolumic relaxation period, defined as the interval from the time of peak negative $\mathrm{d} P / \mathrm{d} t$ to the time when left ventricular pressure fell to $5 \mathrm{mmHg}$ above end-diastolic pressure of the following beat (16). Tau was calculated by three methods. The first used a plot of $1 \mathrm{n}$ left ventricular pressure versus time, as derived by Weiss et al. $(17,18)$. The second used a plot of negative $\mathrm{d} P / \mathrm{d} t$ versus left ventricular pressure, as derived by Raff and Glantz (16). The third method computed the time needed for left ventricular pressure to fall to one-half of its value from peak negative $\mathrm{d} P / \mathrm{d} t$ using the method of Mirsky $\left(\mathrm{T}_{1 / 2}, 19\right)$.

Gated blood pool scintigraphy. Each patient was injected with 0.75 $\mathrm{GBq}(20 \mathrm{mCi})$ of autologous red blood cells labeled in vitro with technetium-99m (20) after left ventriculography. All radionuclide studies were acquired with the patients in the supine position using a mobile Anger camera computer system (model 410, Techicare, Inc., Solon, $\mathrm{OH}$ ) with on-board video image processor computer system. A $30^{\circ}$ slant-hole collinator was used to obtain cephalic angulation in the modified left anterior oblique view. The degree of obliquity varied between $35^{\circ}$ and $45^{\circ}$ and was selected to best visualize the septum. The gated cardiac blood scans were obtained with a $64 \times 64$ matrix for the full field of view $(250 \mathrm{~cm}) .32$ frames per $R R$ interval were acquired. Minimum acquisition time was $3 \mathrm{~min}$, although most studies were acquired for $5 \mathrm{~min}$. The sum of the counts in the 32 frames for the baseline study in the six control patients was 6,748,462 $\pm 1,907,714$. The nonbackground-corrected counts in the left ventricular region of interest at end-diastole was $24,143 \pm 7,963$ and at end-systole was $17,812 \pm 5,892$. The time of each gated study was recorded and a blood sample was obtained at the midpoint of each study. After the baseline scan, repeat scans were obtained at the intermediate and maximum pacing rates.

For each gated study, a ventricular count rate (volume) vs. time curve was obtained with an operator-drawn, fixed left and right ventricular region of interest (21). The operator used the end-diastolic image to identify the septal borders of the ventricles and the strokevolume image to identify the atrial-ventricular and free wall borders of the heart. In patients with left ventricular dysfunction, the end-diastolic image was used to confirm the boundaries of the free wall of the ventricle. Background was derived from computer-generated regions of interest and was assumed to be constant both spatially and temporally.

Since three left ventricular volume versus time curves were obtained in each patient, the relative change in end-diastolic volume between studies was determined by correcting the end-diastolic counts in each curve for acquisition time, physical decay, and biological clearance. Acquisition time for each end-diastolic frame was calculated by multiplying the frame duration ( $R R$ interval/number of frames) by the number of cardiac cycles collected. Loss of counts due to physical decay was corrected for by using the time at which each studied was acquired. Biological clearance of the tracer was calculated by measuring the changes in counts obtained in a well counter of $100-\mu$ l samples of blood obtained at the midpoint of each study. Absolute volumes were obtained by using the baseline left ventricular angiographic enddiastolic volume to calibrate the baseline radionuclide volume.

Left ventricular peak filling rates in each patient were assessed by fitting a third-order polynomial to the rapid diastolic portion of the time-activity curve using a least squares technique and were computed in end-diastolic volumes per second (EDV/s).

The left ventricular peak-systolic pressure-volume ratios were determined for each patient at all pacing rates. These were calculated from the ratio of peak systolic pressure divided by the minimum radionuclide volume $(\mathrm{mmHg} / \mathrm{ml})$.

All radionuclide scans were analyzed by two observers without knowledge of the pacing hemodynamics.

Generation of left ventricular pressure-volume diagrams. Three radionuclide pressure-volume diagrams were recorded in each patient by a method previously described by our laboratory (9). At the midpoint of each modified left anterior oblique scan, left ventricular pressure was recorded. A minimum of six left ventricular pressure curves were then digitized and averaged by a Tektronix 4052 computer (Tektronix Inc., Beaverton, OR). Next, the average left ventricular pressure curve and gated blood pool volume curve were digitized, and pressure-volume diagrams were subsequently plotted from 32 pressurevolume coordinates. A square wave that indicated the time at which the gamma camera's computer system detected the patient's $R$ wave on the electrocardiogram was transmitted to the Electronics-for-Medicine recorder to synchronize the radionuclide volumes with the left ventricular pressure tracings.

Statistics. Mean and standard deviation were calculated for all variables. Multiple groups of data were analyzed by analysis of variance. Paired dimensional data were analyzed by either the paired $t$ test or Wilcoxon signed-rank test. A $P$ value $<0.05$ was considered significant.

\section{Results}

Baseline characteristics of groups A (dilated cardiomyopathy) and B (control) are summarized in Table I. The cardiomyopa- 
Table I. Parameters of Systolic and Diastolic Function at Baseline, Intermediate, and High Atrial Pacing Rates

\begin{tabular}{|c|c|c|c|c|}
\hline Parameter & Group & Baseline & Intermediate & High \\
\hline \multirow[t]{2}{*}{ HR } & A & $86 \pm 11$ & $115 \pm 6^{\ddagger}$ & $141 \pm 6^{\ddagger}$ \\
\hline & B & $74 \pm 8$ & $102 \pm 15^{\ddagger}$ & $134 \pm 12^{\ddagger}$ \\
\hline \multirow[t]{2}{*}{ LVEF } & A & $0.19 \pm 0.11$ & $0.19 \pm 0.11$ & $0.16 \pm 0.09$ \\
\hline & B & $0.69 \pm 0.11$ & $0.68 \pm 0.08$ & $0.68 \pm 0.06$ \\
\hline \multirow{2}{*}{$\mathrm{CO}$} & A & $5.2 \pm 1.6$ & $5.0 \pm 1.5$ & $4.6 \pm 1.3$ \\
\hline & B & $6.4 \pm 1.5$ & $6.1 \pm 1.3$ & $5.3 \pm 1.8$ \\
\hline \multirow[t]{2}{*}{ LVSP } & A & $114 \pm 19$ & $110 \pm 12$ & $107 \pm 15$ \\
\hline & B & $129 \pm 21$ & $126 \pm 15$ & $116 \pm 16$ \\
\hline \multirow[t]{2}{*}{ LVEDP } & $\mathbf{A}$ & $23 \pm 8$ & $18 \pm 9$ & $15 \pm 8$ \\
\hline & B & $13 \pm 3$ & $8 \pm 3$ & $6 \pm 2$ \\
\hline \multirow[t]{2}{*}{ LVEDVI } & $\mathbf{A}$ & $156 \pm 58$ & $170 \pm 51$ & $151 \pm 53$ \\
\hline & B & $81 \pm 17$ & $66 \pm 20$ & $49 \pm 6^{*}$ \\
\hline \multirow[t]{2}{*}{$+\mathrm{d} P / \mathrm{d} t$} & $\mathbf{A}$ & $905 \pm 148$ & $950 \pm 216$ & $945 \pm 244$ \\
\hline & B & $1,305 \pm 185$ & $1,558 \pm 264$ & $1,768 \pm 293^{\ddagger}$ \\
\hline \multirow[t]{2}{*}{$-\mathrm{d} P / \mathrm{d} t$} & $\mathbf{A}$ & $1,624 \pm 224$ & $1,674 \pm 258$ & $1,676 \pm 304$ \\
\hline & B & $2,000 \pm 432$ & $2,137 \pm 360$ & $1,800 \pm 188$ \\
\hline \multirow[t]{2}{*}{$\mathrm{T}_{\ln P \text { vs. } t}$} & $\mathbf{A}$ & $50 \pm 11$ & $51 \pm 12$ & $49 \pm 12$ \\
\hline & B & $40 \pm 9$ & $36 \pm 10$ & $38 \pm 10$ \\
\hline \multirow{2}{*}{$\mathrm{T}_{-\mathrm{d} P / \mathrm{d} t \mathrm{vs} . P}$} & A & $83 \pm 25$ & $71 \pm 25$ & $54 \pm 9^{\ddagger}$ \\
\hline & B & $48 \pm 23$ & $43 \pm 15$ & $39 \pm 8$ \\
\hline \multirow[t]{2}{*}{$T_{1 / 2}$} & $\mathbf{A}$ & $36 \pm 6$ & $37 \pm 7$ & $34 \pm 6$ \\
\hline & B & $29 \pm 8$ & $27 \pm 7$ & $27 \pm 6$ \\
\hline \multirow[t]{2}{*}{ PSP/ESV } & A & $1.1 \pm 0.5$ & $0.9 \pm 0.4$ & $1.0 \pm 0.6$ \\
\hline & B & $4.6 \pm 0.6$ & $6.2 \pm 1.3^{\ddagger}$ & $7.1 \pm 1.0^{\ddagger}$ \\
\hline \multirow[t]{2}{*}{ PFR } & $\mathbf{A}$ & $1.2 \pm 0.5$ & $1.9 \pm 0.8$ & $2.3 \pm 0.7^{*}$ \\
\hline & B & $2.9 \pm 0.9$ & $5.8 \pm 1.5^{\ddagger}$ & $6.5 \pm 0.5^{\ddagger}$ \\
\hline
\end{tabular}

(Group A) Dilated cardiomyopathy; (group B) control. Data are expressed as mean \pm SD. Abbreviations: HR, heart rates (beats/min); LVEF, left ventricular ejection fraction; CO, cardiac output (liter/ $\mathrm{min})$; LVSP, left ventricular systolic pressure ( $\mathrm{mmHg}$ ); LVEDP, left ventricular end-diastolic pressure $(\mathrm{mmHg}), \mathrm{LVEDVI}$, left ventricular end-diastolic volume index $\left(\mathrm{ml} / \mathrm{m}^{2}\right) ;+\mathrm{d} P / \mathrm{d} t$, peak positive $\mathrm{d} P / \mathrm{d} t$ $(\mathrm{mmHg} / \mathrm{s}),-\mathrm{d} P / \mathrm{d} t$, peak negative $\mathrm{d} P / \mathrm{d} t(\mathrm{mmHg} / \mathrm{s}), \mathrm{T}_{\ln P \text { va }, t}$, tau as derived by Weiss et al. $(19,20)$; $T_{-d P / d t}$ vs. $P$, tau as derived by Raff and Glantz (18); $T_{1 / 2}$, tau using the method of Mirsky (21); PSP/ ESV, the ratio of peak systolic pressure to end systolic volume; PFR, peak filling rate $(E D V / s)$

${ }^{*} P \leq 0.05 ;{ }^{\ddagger} P<0.01$.

thy group had a left ventricular ejection fraction of $0.19 \pm 0.11$, a left ventricular end-diastolic volume index of $156 \pm 58$ $\mathrm{ml} / \mathrm{m}^{2}$, and a left ventricular end-diastolic pressure of $23 \pm 8$ $\mathrm{mmHg}$. The control group had a left ventricular ejection fraction of $0.69 \pm 0.11$, an end-diastolic volume index of $81 \pm 17$ $\mathrm{ml} / \mathrm{m}^{2}$, and a left ventricular end-diastolic pressure of $13 \pm 3$ $\mathrm{mmHg}$.

Pacing-induced hemodynamics (Table I). There was no difference between the baseline or maximum heart rates to which groups A and B were paced. Moreover, pacing resulted in no significant change in cardiac output or left ventricular systolic pressure in either group. Left ventricular end-diastolic pressure declined progressively in both groups during pacing tachycardia, but the extent of fall in pressure was less in the cardiomyopathy group ( $34 \%$ of baseline), than in the control group (52\% of baseline, $P<0.05$ ). Peak positive $\mathrm{d} P / \mathrm{d} t$ was unchanged in the cardiomyopathy group (905 \pm 148 to $945 \pm 244, P=$ NS) but increased in control patients at in-

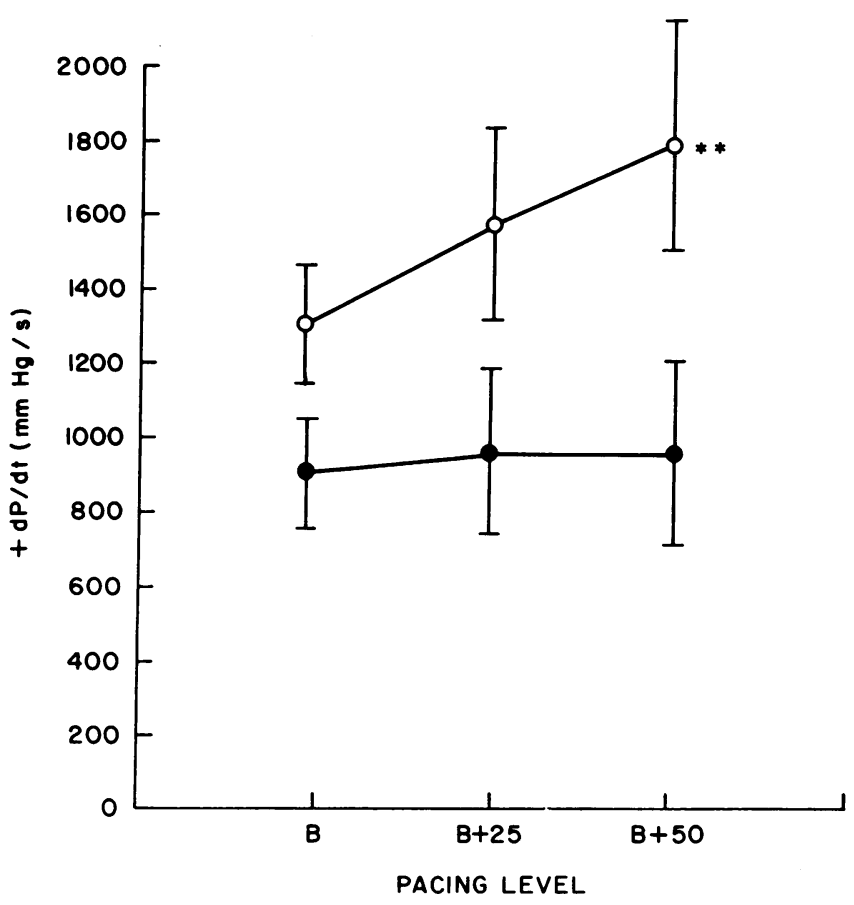

Figure 1. Changes in peak positive $\mathrm{d} P / \mathrm{d} t$ in response to atrial pacing tachycardia. (0) Control; (•) cardiomyopathy; (**) $P<0.01$. B, baseline heart rate; $B+25$, baseline heart rate plus 25 beats $/ \mathrm{min} ; B+50$, baseline heart rate plus 50 beats $/ \mathrm{min}$.

creasing heart rate $(1,305 \pm 185$ to $1,768 \pm 293, P<0.005$, Fig. 1). Peak negative $\mathrm{d} P / \mathrm{d} t$ was unchanged in both the cardiomyopathy $(1,624 \pm 224$ to $1,676 \pm 304, P=N S)$ and control patients $(2,000 \pm 432$ to $1,800 \pm 188, P=N S)$. Tau, as determined by a plot of $\ln P$ vs. time and the method of Mirsky, did not change with increasing heart rate in either the cardiomyopathy or control groups. There was a decrease in tau, however, as determined by a plot of negative $\mathrm{d} P / \mathrm{d} t$ vs. left ventricular pressure in the cardiomyopathy group $(83 \pm 25$ to $54+9 \mathrm{~ms}, P$ $<0.01$ ) while a similar trend in the control group did not achieve statistical significance ( $48 \pm 23$ to $39 \pm 8 \mathrm{~ms}, P=\mathrm{NS})$ in response to increasing heart rate.

Radionuclide ventriculography. The left ventricular ejection fraction did not change in either group during pacing tachycardia. The cardiomyopathy group left ventricular ejection fraction was $0.19 \pm 0.11$ at baseline, and $0.16 \pm 0.09$ at peak pacing $(P=\mathrm{NS})$. The control group left ventricular ejection fraction was $0.60 \pm 0.11$ at baseline, and $0.68 \pm 0.06$ at peak pacing $(P=\mathrm{NS})$.

Left ventricular end-diastolic volumes were considerably greater in the cardiomyopathy patients $\left(156 \pm 58 \mathrm{ml} / \mathrm{m}^{2}\right)$ than in the control patients $\left(81 \pm 17 \mathrm{ml} / \mathrm{m}^{2}, P<0.05\right)$. The end-diastolic volume did not change with increasing heart rate in the cardiomyopathy group ( $156 \pm 58$ to $\left.151 \pm 53 \mathrm{ml} / \mathrm{m}^{2}, P=\mathrm{NS}\right)$. However, there was a decrease of $38 \%$ in the end-diastolic volume of the control patients at peak pacing ( $81 \pm 17$ to $49 \pm 6$ $\mathrm{ml} / \mathrm{m}^{2}, P<0.05$, Fig. 2).

Left ventricular peak filling rate changed slightly with pacing tachycardia in the cardiomyopathy group (1.2 \pm 0.5 to $2.3 \pm \mathrm{EDV} / \mathrm{s}, P=0.05)$, but increased in the control group $(2.9 \pm 0.9$ to $6.5 \pm 0.5 \mathrm{EDV} / \mathrm{s}, P<0.001$, Fig. 3$)$.

Pressure-volume diagrams. The serial changes in pressurevolume diagrams of the seven cardiomyopathy patients are shown in Fig. 4, with a more detailed presentation of the dia- 


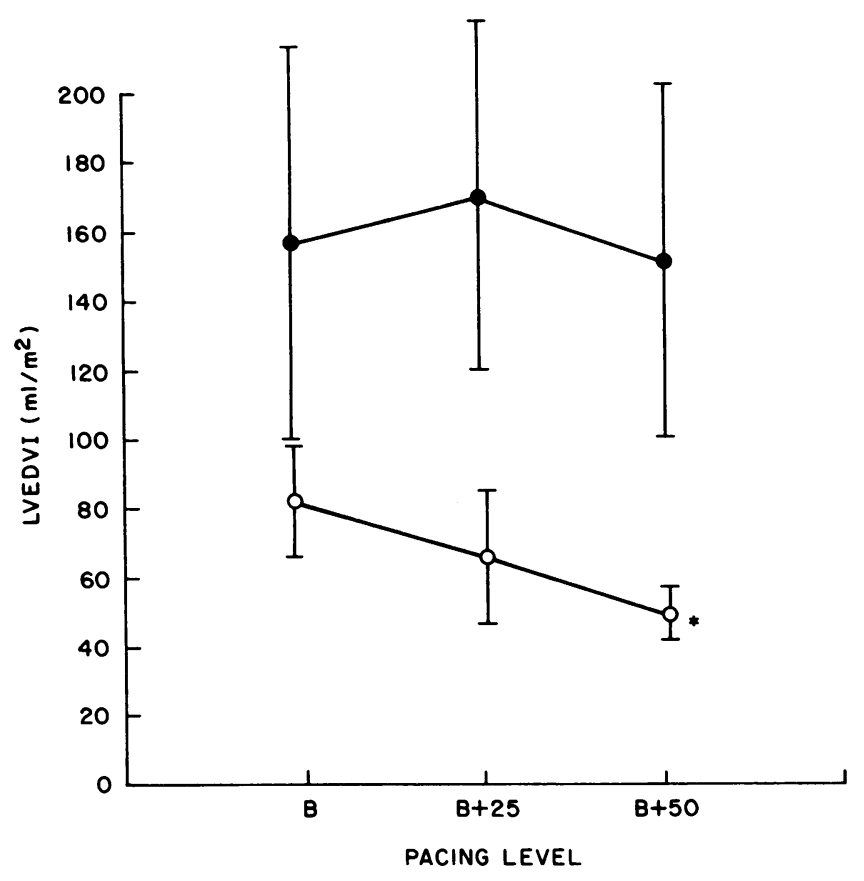

Figure 2. Changes in left ventricular end-diastolic volume index (LVEDVI) in response to atrial pacing tachycardia. (0) Control; (•) cardiomyopathy; (*) $P<0.05$. B, baseline heart rate; $\mathrm{B}+25$, baseline heart rate plus 25 beats/min; B +50 , baseline heart rate plus 50 beats/min.

stolic limbs of these same patients in Fig. 5. There is little to no leftward shift of the end-systolic pressure-volume relationship. Specifically, patients B and C had mild leftward displacement, patients $A$ and $E$ had mild rightward displacement, and patients $D, F$, and $G$ had marked rightward and downward displacement of the end-systolic pressure-volume relationship. There is minimal to no downward shift of the diastolic pressure-volume relationship, except a mild rightward shift for patient $A$. In comparison, the pressure-volume diagrams for the six control patients are shown in Fig. 6, with a more detailed presentation of the diastolic limbs of these same patients in Fig. 7. There is a progressive leftward shift for the end-systolic pressure-volume relationship, and a progressive downward diastolic shift.

The ratio of peak-systolic pressure to end-systolic volume did not change in patients with cardiomyopathy $(1.1 \pm 0.5$ to $1.0 \pm 0.6, P=\mathrm{NS})$, but increased with each pacing rate in the controls (4.6 \pm 0.6 to $7.1 \pm 1.0, P<0.001$, Fig. 8 ).

\section{Discussion}

This study has documented that the enhancement of systolic and diastolic function may be blunted in patients with dilated cardiomyopathy in response to pacing tachycardia compared to controls. These findings suggest a depression of both inotropic and lusitropic reserve. The lack of improvement in isovolumic phase indices (e.g., peak-positive $\mathrm{d} P / \mathrm{d} t$ ) and end-systolic contractile indices (e.g., peak-systolic pressure end-systolic volume ratio) in cardiomyopathy patients has been contrasted to patients with normal ventricular function who demonstrated significant increases in both of these parameters. Moreover, the absence of a leftward shift of the pressure-volume diagram in cardiomyopathy patients is sharply contrasted to patients

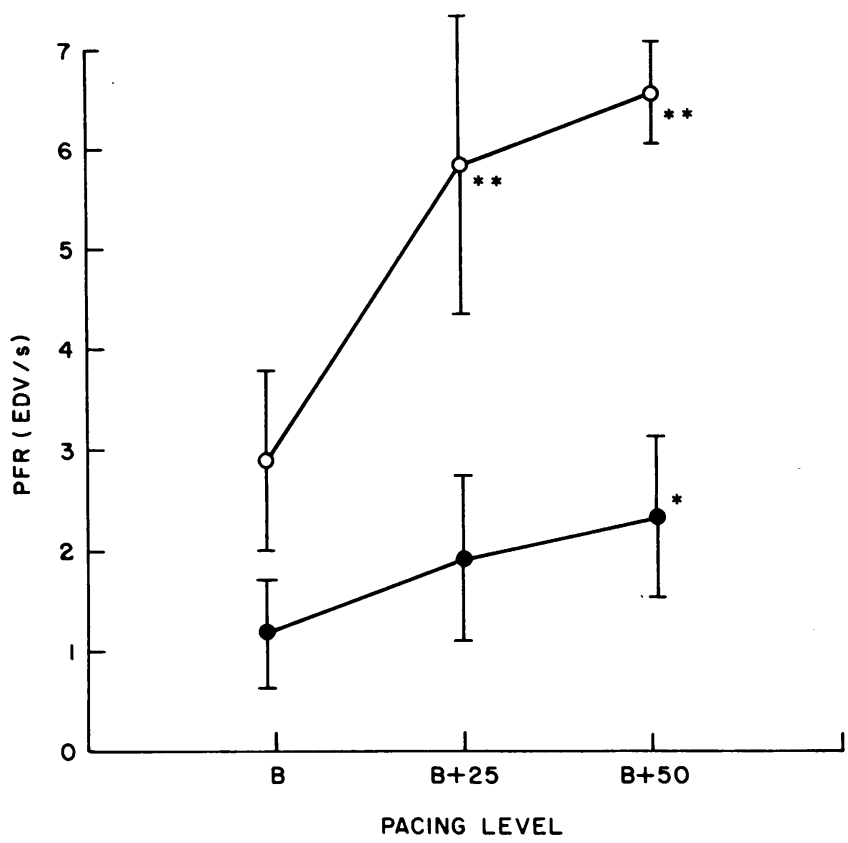

Figure 3. Changes in peak filling rate (PFR) in response to atrial pacing tachycardia. (O) Control; (•) cardiomyopathy; (*) $P \leq 0.05$; (**) $P$ $<0.01$. B, baseline heart rate; $B+25$, baseline heart rate plus 25 beats $/ \mathrm{min} ; \mathrm{B}+50$, baseline heart rate plus 50 beats $/ \mathrm{min}$.

with normal left ventricular function who demonstrated progressive decreases in ventricular volume with increasing heart rate. In terms of diastolic function, cardiomyopathy patients demonstrated a minimal increase in left ventricular peak filling rate, in contrast to the marked increase in ventricular filling in control patients. In addition, the blunted or absent downward shift of the diastolic portion of the pressure-volume diagram of cardiomyopathy patients is different from the progressive downward shift in patients with normal left ventricular function.

Chronotropic changes in patients with normal left ventricular function. Over a century has passed since Bowditch described the "staircase" or "Treppe" phenomenon, referring to the increase in myocardial contractility associated with faster rates of contraction (1). Woodworth, in 1902, confirmed both ascending and descending limbs of the staircase effect (2). In addition, he extended this phenomenon to spontaneously occurring extra contractions, demonstrating that the beat after a compensatory pause has increased contractility. Numerous laboratories have documented this same phenomenon with either increased contraction frequency or paired electrical stimulation in isolated papillary muscle $(3,4,13)$, in situ hearts of laboratory animals $(8,12,22,23)$, and intact hearts in humans (24-26).

Studies in isolated papillary muscles have provided some insight into the mechanism of the Treppe effect. Increased frequency of stimulation or paired-stimulation both produce (a) increase in the maximum rate of tension development, $(b)$ increase in the peak tension developed, and $(c)$ shortening of the duration of the active state. The increase in maximum rate of tension development reflects enhanced contractility, as demonstrated by shifting the force-velocity relationship upward and to the right (13). Opposing the maximum rate of 


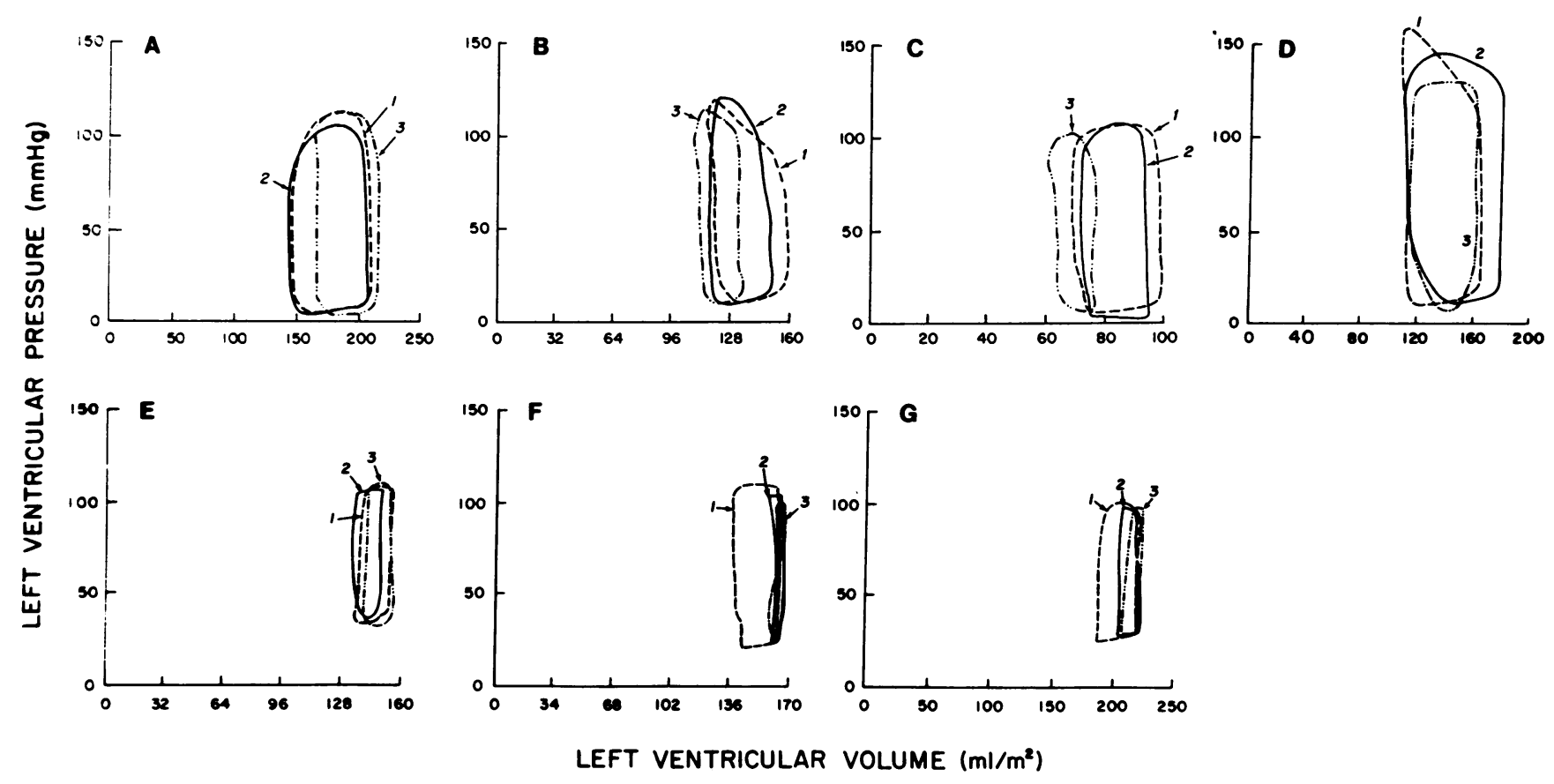

Figure 4. Sequential pressure-volume diagrams with pacing tachycardia in the seven patients $(A-G)$ with dilated cardiomyopathy are shown. Diagram 1 (dashed lines) is the baseline pressure-volume relationship, and diagrams 2 (solid lines) and 3 (dots and dashes) intermediate and high atrial pacing, respectively.
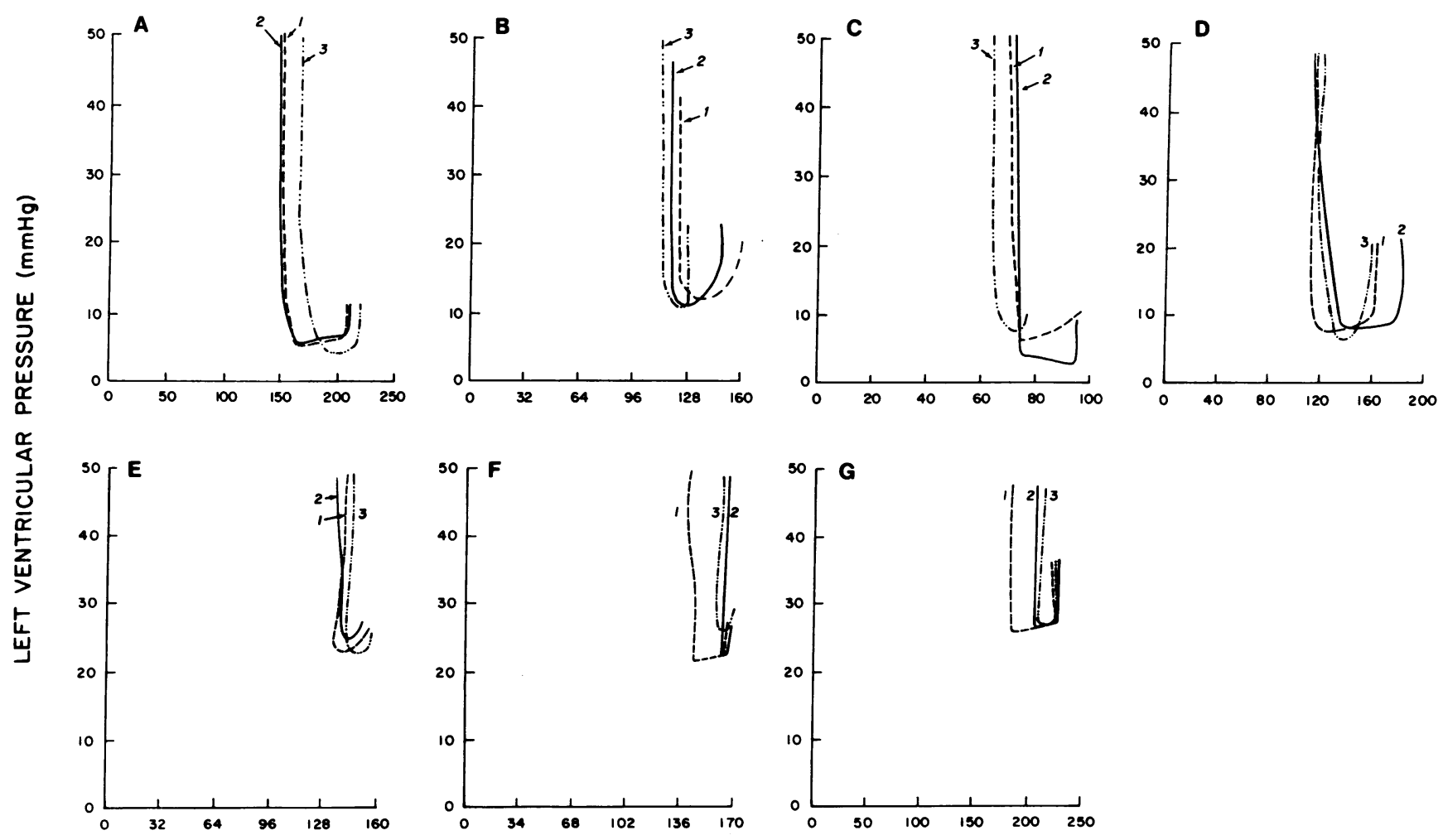

\section{LEFT VENTRICULAR VOLUME $\left(\mathrm{ml} / \mathrm{m}^{2}\right)$}

Figure 5. The diastolic limb of sequential pressure-volume diagrams with pacing tachycardia in the seven patients $(A-G)$ with dilated cardiomyopathy are shown. Diastolic limb 1 (dashed lines) is the baseline pressure-volume relationship, and diastolic limbs 2 (solid lines) and 3 (dots and dashes) intermediate and high atrial pacing rates, respectively. The entire pressure-volume diagram for these same patients is depicted in Fig. 4. 

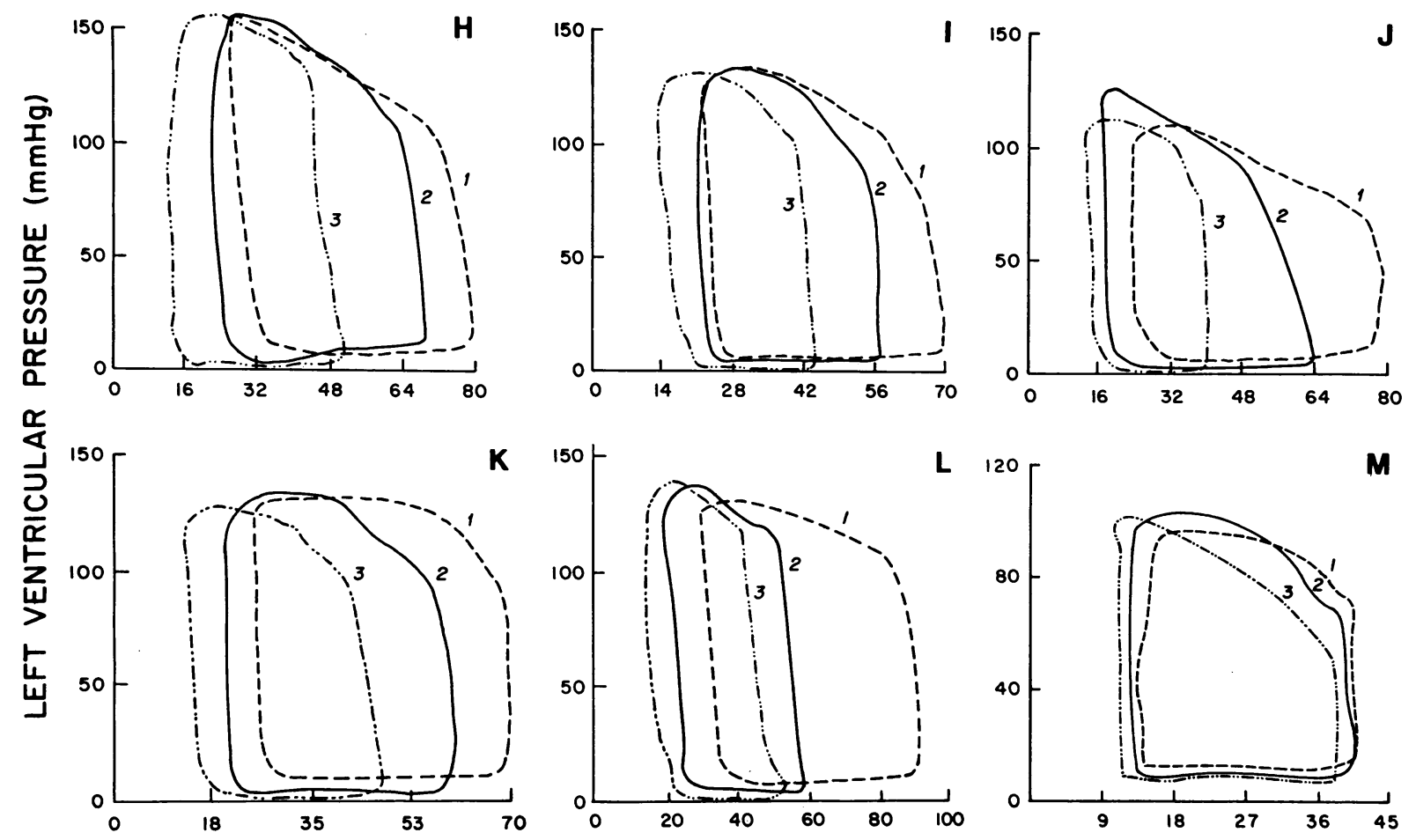

\section{LEFT VENTRICULAR VOLUME $\left(\mathrm{ml} / \mathrm{m}^{2}\right)$}

Figure 6. Sequential pressure-volume diagrams with pacing tachycardia in the six patients $(H-M)$ with normal left ventricular function are shown. Diagram 1 (dashed lines) is the baseline pressure-volume relationship, and diagrams 2 (solid lines) and 3 (dots and dashes) intermediate and high atrial pacing, respectively. Patient diagrams $K$ and $L$ have previously been published (references 10 and 9 , respectively).

tension development is shortening of the duration of the active state. Increasing peak tension occurs at greater frequency of stimulation because the increase in maximum rate of tension development more than offsets the effect of decreasing the duration of the active state. All the above-mentioned effects have been demonstrated in in situ animal models of congestive heart failure. In fact, the effects of paired-stimulation have been most evident in the failing ventricle and least marked in the normal heart (12). Unfortunately, application of paired stimulation has not been found beneficial in patients with heart failure $(14,15)$.

Evidence of the existence of the Treppe effect in man has been documented utilizing isovolumetric and ejection phase indices of contractility in humans without heart failure. DeMaria et al. (24) and Krayenbuehl et al. (25) both documented decreased ventricular volumes, and increased velocity indices and fractional shortening in response to atrial pacing in the catheterization laboratory. Ricci et al. (26) extended these findings and documented increasing stroke volume and velocity of circumferential fiber shortening from the first to late tachycardia beats. To exclude reflex release of catecholamines, they repeated these studies in cardiac transplant recipients and similar results were found.

Our laboratory has previously extended these findings to end-systolic contractile parameters $(9,10)$. The end-systolic pressure-volume point, and in several patients where loading conditions were altered, the end-systolic pressure-volume line, were shifted to the left and upward in response to atrial pacing. In the present study, we reconfirm these findings. Patients with normal left ventricular function demonstrated an increase in peak positive $\mathrm{d} P / \mathrm{d} t$, an increase in the peak systolic pressure to end-diastolic volume ratio, and a leftward shift of the end-systolic pressure volume point in response to atrial pacing tachycardia.

Improvement in parameters of diastolic left ventricular function during pacing tachycardia have also been noted. Karliner et al. (11) and Grossman et al. (27) demonstrated tachycardia-related increases in peak negative $\mathrm{d} P / \mathrm{d} t$. A decrease in the time constant of left ventricular relaxation (tau, 17) has been described. Our laboratory demonstrated, in an earlier study, a tachycardia-related downward shift of the diastolic limb of the pressure-volume diagram and increase in the peak filling rate in normal subjects (10). In our current study, we again show that atrial pacing-tachycardia in control subjects is associated with an increase in peak filling rate and downward shift of the diastolic limb of the pressure-volume diagram, with falls in both pressure and volume. Tau, however, did not decrease significantly, although a downward trend was evident using the method of Raff et al. (16).

Chronotropic changes in patients with heart failure. There has been little investigation of the influence of heart rate on systolic and diastolic function in patients with primary myocardial failure. Grossman et al. (27) demonstrated a depressed peak negative $\mathrm{d} P / \mathrm{d} t$ at baseline and no increase in the velocity of circumferential fiber lengthening or shortening during atrial pacing in patients with dilated cardiomyopathy compared to controls. Erbel et al. (28) found no change in left ventricular end-systolic or end-diastolic volumes and a significant reduction in left ventricular ejection fraction during atrial pacing tachycardia in patients with dilated cardiomyopathy compared to controls. The present study is in agreement with these earlier observations and extends them by examining end-sys- 

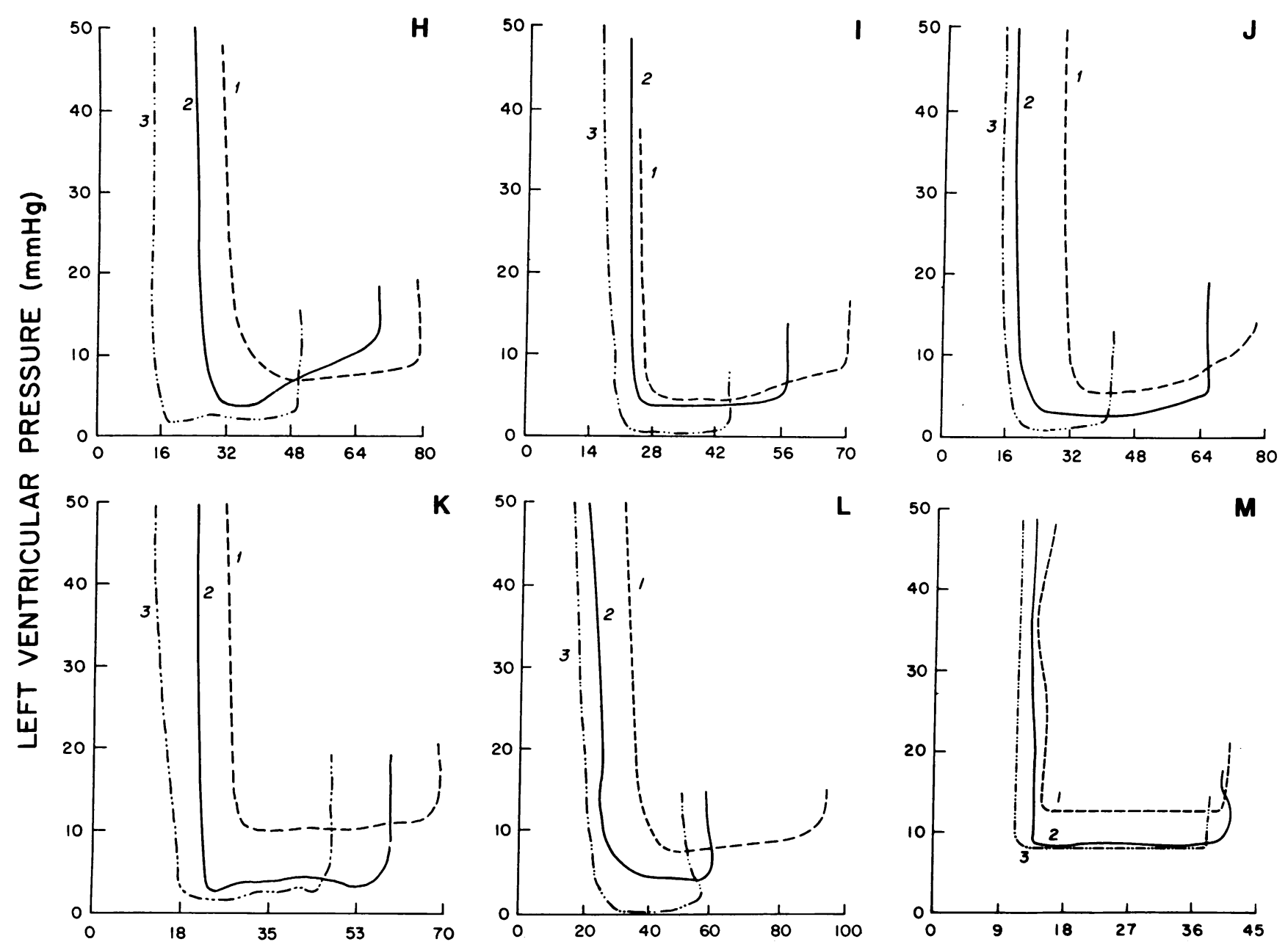

\section{LEFT VENTRICULAR VOLUME $\left(\mathrm{ml} / \mathrm{m}^{2}\right)$}

Figure 7. The diastolic limb of sequential pressure-volume diagrams with pacing tachycardia in the six patients $(H-M)$ with normal left ventricular function are shown. Diastolic limb 1 (dashed lines) is the baseline pressure-volume relationship, and diastolic limbs 2 (solid

tolic pressure-volume indices of contractile function as well as radionuclide peak filling rate and the left ventricular diastolic pressure-volume relationship.

The physiologic mechanism explaining the lack of a Treppe effect and absence of improved diastolic function with tachycardia in patients with left ventricular failure has yet to be defined. Wood et al. (29) demonstrated that increased depolarization of the sarcolemma during the plateau phase of the cardiac action potential promoted calcium entry by way of slow calcium channels. Paired stimulation and increasing frequency of stimulation may also provide increased calcium availability to the myofilaments. Proof of calcium involvement has been provided in recent studies by Morgan and Blinks (30) using aequorin, a bioluminescent protein which acts as a calcium tag. Increased pacing rates of papillary muscles produced increased peak tension, and a parallel increase in the aequorin signal. Similar observations have been made by Wier and Yue (31).

Disturbed calcium handling may blunt the expected increase in contractility in response to tachycardia. Several investigators have demonstrated depressed calcium release by lines) and 3 (dots and dashes) intermediate and high atrial pacing rates, respectively. The entire pressure-volume diagram for these same patients is depicted in Fig. 6.

the sarcoplasmic reticulum in failing human myocardium (32, 33). Gwathmey et al. (34) have noted a second component to the aequorin signal in myocardium from patients undergoing transplantation, perhaps representing delayed calcium uptake by the sarcoplasmic reticulum. Diastolic relaxation of the myocardium is dependent on removal of calcium from the myofilaments and several reports have documented slower rates of calcium uptake by sarcoplasmic reticulum from failing cardiac tissue (32-37). Hence, the shortened duration of diastole during tachycardia coupled with the slowed rate of calcium release and uptake by the sarcoplasmic reticulum in the failing myocardium may account for the absence of improvement in parameters of systolic and diastolic function during pacing tachycardia in patients with dilated cardiomyopathy.

An alternative explanation would be ischemia. Two cardiomyopathy patients had mild rightward and three had marked rightward and downward displacement of the end-systolic pressure-volume relationship in response to pacing tachycardia. In addition, one cardiomyopathy patient had a mild rightward shift of the diastolic limb of the pressure-volume relationship. Patients with cardiomyopathy have in- 


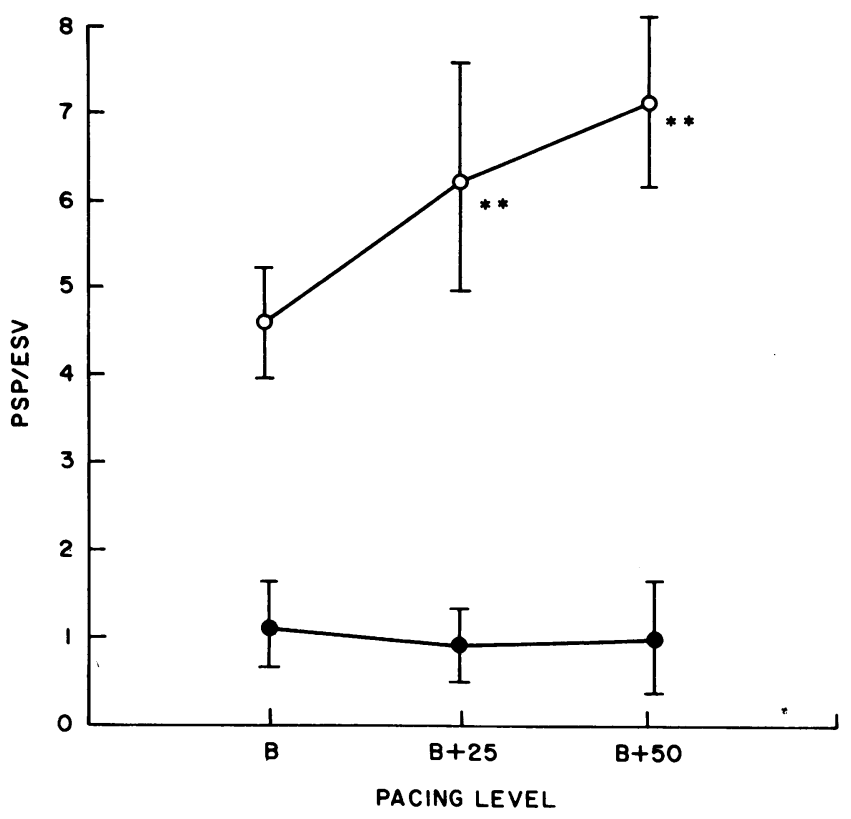

Figure 8. Changes in the ratio of peak systolic pressure (PSP) to endsystolic volume. (ESV). (O) Control; (•) cardiomyopathy; (**) $P$ $<0.01$. B, baseline heart rate; $B+25$, baseline heart rate plus 25 beats $/ \mathrm{min} ; \mathrm{B}+50$, baseline heart rate plus 50 beats $/ \mathrm{min}$.

creased left ventricular mass and myocyte hypertrophy. Hypertrophied cardiac muscle has an increased vulnerability to ischemia $(38,39)$. It is possible that pacing induced ischemia was responsible for the alterations in the pressure-volume relationships mentioned above rather than abnormal calcium handling.

Limitations of study. Left ventriculography was performed with ionic contrast-enhancement medium before the pacing protocol was initiated. It is possible that the depressant effect of the contrast agent on left ventricular function was more profound in the cardiomyopathy group compared to patients with normal left ventricular function, and partially explain the diminished positive inotropic and lusitropic effects of atrial pacing in the cardiomyopathy group. $30 \mathrm{~min}$, however, elapsed between administration of ionic contrast medium, and the pacing protocol. A second concern is baroreceptor dysfunction in heart failure. Both patients with cardiomyopathy and normal ventricular function had a fall in left ventricular systolic pressure with pacing, although these changes did not reach statistical significance (Table I). In normal subjects, a fall in systolic arterial pressure is accompanied by activation of the sympathetic nervous system with a subsequent increase in heart rate (40). In contrast, subjects with cardiomyopathy usually do not demonstrate significant changes in circulating catecholamines in response to hypotension (40). If reflex sympathetic stimulation was elicited by pacing in our study, a rise in circulating catecholamines in the patients with normal ventricular function might partially explain our results. Atrial pacing in our normal patient population, however, resulted in no increase in post-pacing heart rate $(74 \pm 8$ to $77 \pm 16, P$ $>0.05$ ) arguing against elicitation of sympathetic stimulation. A third concern is the continuation of cardiac medications up until the time of cardiac catheterization. All seven patients with congestive heart failure were receiving digoxin. KochWeser and Blinks (41) abolished the positive Treppe effect in mammalian papillary muscle by increasing extracellular cal- cium, or with the use of cardiac glycosides. The latter, via inhibition of the $\mathrm{Na}^{+}-\mathrm{K}^{+}$pump, increases intracellular calcium. Digoxin could have contributed to the depressed Treppe effect seen in the cardiomyopathic patients. Five of the six normal patients were receiving calcium-channel blocking agents. It is unlikely, however, that these medications enhanced the Treppe effect. Applegate et al. (42) demonstrated no change or depression of peak positive $\mathrm{d} P / \mathrm{d} t$ in response to pacing in the presence of calcium channel blockade.

Conclusion. In patients with normal left ventricular function, pacing tachycardia represents a positive inotropic stimulus as evidenced by an increase in peak positive $\mathrm{d} P / \mathrm{d} t$, an increase in the peak-systolic pressure to end-diastolic volume ratio, and a leftward shift of the end-systolic pressure-volume relationship. These increases in contractile indices are absent or diminished in patients with idiopathic dilated cardiomyopathy. In addition, normal left ventricles showed improvement in diastolic function during tachycardia, with increasing peak filling rate and a downward shift of the diastolic limb of the pressure-volume diagram. This improvement of diastolic function is blunted in patients with dilated cardiomyopathy, where no change in peak filling rate and minimal or no downward shift of the diastolic limb of the pressure-volume diagram occurred. The lack of a Treppe effect in patients with dilated cardiomyopathy suggests an insufficiency of contractile reserve, possibly secondary to impaired intracellular calcium release. Likewise, improvement in diastolic parameters during tachycardia may also be blunted in patients with dilated cardiomyopathy, possibly due to impaired calcium reuptake.

\section{Acknowledgments}

This study was supported in part by grant HL07374 from the National Heart, Lung, and Blood Institute.

\section{References}

1. Bowditch, H. P. 1871. Über die Eigenthumlichkeiten der Reizbarkeit, welche die Muskelfasern des Herzens Zeiger Verh. K. Sachs. Ges. Wiss. Leipzig. 23:652-689.

2. Woodworth, R. S. 1902. Maximal contraction, "staircase" contraction, refractory period and compensatory pause of the heart. Am.J. Physiol. 8:213-249.

3. Koch-Weser, J. 1963. Effect of rate changes on strength and time course of contraction of papillary muscle. Am. J. Physiol. 204:451457.

4. Blinks, J. R., and J. Koch-Weser. 1961. Analysis of the effects of changes in rate and rhythm upon myocardial contractility. J. Pharmacol. 134:373-389.

5. Smith, V. E., and A. M. Katz. 1983. Inotropic and lusitropic abnormalities in the genesis of heart failure. Eur. Heart J. 4(Suppl. A):7-17.

6. Gleason, W. L., and E. Braunwald. 1962. Studies on the first derivative of the ventricular pressure pulse in man. J. Clin. Invest. 41:80-91.

7. Barnes, G. E., L. D. Horwitz, and V. S. Bishop. 1979. Reliability of the maximum derivatives of left ventricular pressure and internal diameter as indices of the inotropic state of the depressed myocardium. Cardiovasc. Res. 13:652-662.

8. Mitchell, J. H., A. G. Wallace, and N. S. Skinner. 1963. Intrinsic effects of heart rate on left ventricular performance. Am. J. Physiol. 205:41-48.

9. McKay, R. G., J. M. Aroesty, G. V. Heller, H. Royal, J. A. Parker, K. J. Silverman, G. M. Kolodny, and W. Grossman. 1984. Left ventricular pressure-volume diagrams and end-systolic pressure-volume relations in human beings. J. Am. Coll. Cardiol. 3:301-312. 
10. Aroesty, J. M., R. G. McKay, G. V. Heller, H. D. Royal, A. Als, and W. Grossman. 1985. Simultaneous assessment of left ventricular systolic and diastolic dysfunction during pacing-induced ischemia. Circulation. 71:889-900.

11. Karliner, J. S., M. M. LeWinter, F. Mahler, R. Engler, and R. A. O'Rourke. 1976. Pharmacological and hemodynamic influences on the rate of isovolumetric left ventricular relaxation in the conscious dog. J. Clin. Invest. 58:751-760.

12. Hoffman, B. F., H. J. Bartelstone, B. J. Scherlag, and P. F. Cranefield. 1965. Effects of post extrasystolic potentiation on normal and failing hearts. Bull. N.Y. Acad. Med. 41:498-534.

13. Sonnenblick, E., P. L. Frommer, and E. Braunwald. 1965. Electroaugmentation of human and cat papillary muscle produced by paired electric stimulation. Bull. N.Y. Acad. Med. 41:554-559.

14. Resnekov, L., E. Sowton, P. Lord, and J. Norman. 1966. Haemodynamic and clinical effects of paired stimulation of the heart. $\mathrm{Br}$. Heart J. 28:622-630.

15. Braunwald, E., J. Ross, E. H. Sonnenblick, P. L. Frommer, N. S. Braunwald, and A. G. Morrow. 1965. Slowing of heart rate, electroaugmentation of ventricular performance, and increase of myocardial oxygen consumption produced by paired electrical stimulation. Bull. N.Y. Acad. Med. 41:481-497.

16. Raff, G. L., and S. A. Glantz. 1981. Volume loading slows left ventricular isovolumic relaxation rate: evidence of load-dependent relaxation in the intact dog heart. Circ. Res. 48:813-824.

17. Weiss, J. L., J. W. Frederiksen, and M. L. Weisfeldt. 1976. Hemodynamic determinants of the time-course of fall in canine left ventricular pressure. J. Clin. Invest. 58:751-760.

18. Frederiksen, J. W., J. L. Weiss, and M. L. Weisfeldt. 1978. Time constant of isovolumic pressure fall: determinants in the working left ventricle. Am. J. Physiol. 235:H701-H706.

19. Mirsky, I. 1984. Assessment of diastolic function: suggested methods and future considerations. Circulation. 69:836-841.

20. Richards, P., and T. D. Smith. 1976. A simple kit for the preparation of technetium $99 \mathrm{~m}$-labeled red blood cells. J. Nucl. Med. 17:126-132.

21. Holman, B. L., J. Wynne, J. S. Zielonka, and J. D. Idoine. 1981. A simplified technique for measuring right ventricular ejection fraction using equilibrium radionuclide angiocardiograms and the slant-hole collinator. Radiology. 138:429-436.

22. Chardack, W. M., A. A. Gage, and D. C. Dean. 1965. Paired and coupled electrical stimulation of the heart. Bull. N.Y. Acad. Med. 41:462-480.

23. Katz, L. N. 1965. Effects of artificially induced paired and coupled beats. Bull. N.Y. Acad. Med. 41:428-461.

24. DeMaria, A., A. Neuman, D. Schubart, G. Lee, and D. Mason. 1979. Systematic correlation of cardiac chamber size and ventricular performance determined with echocardiogram and alternations in heart rate in normal persons. Am. J. Cardiol. 43:1-14.

25. Krayenbuehl, H., M. Schoenbeck, W. Rutishauser, and P. Winz. 1975. Abnormal segmental contraction velocity in coronary artery disease produced by isometric exercise and atrial pacing. Am. J. Cardiol. 35:785-794.

26. Ricci, D. R., A. E. Orlick, E. L. Alderman, N. B. Ingels, G. T. Daughters, C. A. Kusnick, B. A. Reitz, and E. B. Stinon. 1979. Role of tachycardia as an inotropic stimulus in man. J. Clin. Invest. 63:695703.

27. Grossman, W., L. P. McLaurin, and E. L. Rolett. 1979. Alteration in left ventricular relaxation and diastolic compliance in congestive cardiomyopathy. Cardiovasc. Res. 13:514-522.

28. Erbel, R., P. Schweizer, W. Krebs, H. Langen, J. Meyer, and S. Effert. 1984. Effects of heart rate changes on left ventricular volume and ejection fraction: a two-dimensional echocardiographic study. Am. J. Cardiol. 53:590-597.

29. Wood, E. H., R. L. Hepner, and S. Weidmann. 1969. Inotropic effects of electric currents. Circ. Res. 24:409-445.

30. Morgan, J. P., and J. R. Blinks. 1982. Intracellular calcium transients in the cat papillary muscle. Can. J. Physiol. Pharmacol. 60:524-528.

31. Wier, W. G., and D. T. Yue. 1986. Intracellular calcium transients underlying the short-term force-interval relationship in ferret ventricular myocardium. J. Physiol (Lond.). 376:507-530.

32. Harigaya, S., and A. Schwartz. 1969. Rate of calcium binding and uptake in normal animal and failing human cardiac muscle. Circ. Res. 25:781-794.

33. Lindenmayer, G. E., L. A. Sordahl, S. Harigaya, J. C. Allen, H. R. Besch, and A. Schwartz. 1971. Some biochemical studies on subcellular systems isolated from fresh recipient human cardiac tissue obtained during transplantation. Am. J. Cardiol. 27:277-283.

34. Gwathmey, J. K., L. Copelas, R. MacKinnon, F. J. Schoen, M. D. Feldman, W. Grossman, and J. P. Morgan. 1987. Abnormal intracellular calcium handling in myocardium from patients with end-stage heart failure. Circ. Res. 61:70-76.

35. Gertz, E. W., M. L. Hess, R. F. Lain, and F. N. Briggs. 1967. Activity of the vesicular calcium pump in the spontaneously failing heart-lung preparation. Circ. Res. 20:477-484.

36. Suko, J., J. H. K. Vogel, and C. A. Chidsey. 1970. Intracellular calcium and myocardial contractility. 3. Reduced calcium uptake and ATPase of the sarcoplasmic reticular fraction prepared from chronically failing calf hearts. Circ. Res. 27:235-247.

37. Sulakhe, P. V., and N. S. Dhalla. 1971. Excitation-contraction coupling in the heart. 7. Calcium accumulation in subcellular particles in congestive heart failure. J. Clin. Invest. 50:1019-1027.

38. Bache, R. J., C. E. Arentzen, A. B. Simon, and T. R. Vrobel. 1984. Abnormalities in myocardial perfusion during tachycardia in dogs with left ventricular hypertrophy: metabolic evidence for myocardial ischemia. Circulation. 69:409-417.

39. Alyono, D., R. W. Anderson, D. G. Parrish, X. Dai, and R. J. Bache. 1986. Alterations of myocardial blood flow associated with experimental canine left ventricular hypertrophy secondary to valvular aortic stenosis. Circ. Res. 58:47-57.

40. Hirsch, A. T., V. J. Dzau, and M. A. Creager. 1987. Baroreceptor function in congestive heart failure: effect of neurohumoral activation and regional vascular resistance. Circulation. 75(Suppl. IV):3648.

41. Koch-Weser, J., and J. R. Blinks. 1963. The influence of the interval between beats on myocardial contractility. Pharmacol. Rev. 15:601-652.

42. Applegate, R. J., R. A. Walsh, and R. A. O'Rourke. 1987. Frequency-dependent myocardial depression induced by verapamil in conscious dogs. Am. J. Physiol. 253:H487-492. 